\title{
Abhandlung
}

Dominik Brodowski, LL.M. (UPenn)

\section{Anonyme Ehrverletzungen in Internetforen: Ein Lehrstück strafrechtlicher (Fehl-)Regulierung}

\author{
Dominik Brodowski: Der Autor ist Wiss. Mitarbeiter am Lehrstuhl \\ für Strafrecht, Strafprozessrecht und Wirtschaftsstrafrecht \\ (Prof. Dr. Joachim Vogel, RiOLG) der LMU München
}

Ein Durchsuchungsbeschluss gegen die Augsburger Allgemeine Zeitung, um die Identität des Benutzers »berndi« aufzudecken, durch dessen Äußerung im zeitungseigenen Internetforum sich der Ordnungsreferent der Stadt Augsburg beleidigt fühlte, ${ }^{1}$ und die Anordnung von Beugehaft gegen einen Onlineredakteur eines Klinik-Bewertungsportals, um die Identität des Urhebers einer mutmaßlich üblen Nachrede aufzudecken, ${ }^{2}$ sorgten nicht nur in der Internetöffentlichkeit für Aufsehen. Dieser Beitrag erörtert, ob anonyme Äußerungen einen geringeren verfassungsrechtlichen Schutz

$1 \mathrm{Vgl}$. die Entscheidung über die hiergegen eingelegte Beschwerde, LG Augsburg, Beschl. v. 19. 3. 2013 - 1 Qs 151/13, in diesem Heft S. 536.

2 Vgl. Lorenz/Huff LTO v. 19. 2. 2013, verfügbar unter http://www. lto.de/persistent/a_id/8174/ (Stand: 24. 5. 2013). genießen sowie in welchem Umfang presserechtliche Beschlagnahmeverbote und Zeugnisverweigerungsrechte einer Identifizierung des Autors einer ehrverletzenden Äußerung im Internet entgegenstehen. Dabei zeigt sich, dass das Internetstrafrecht in seiner vorherrschenden aber fehlerhaften Auslegung Fehlanreize setzt, da sie denjenigen Forenbetreiber dem Risiko einer Bestrafung aussetzt, der freiwillig Beiträge vor ihrer Onlinestellung kontrolliert.

Das Internet - insbesondere in seiner Ausgestaltung als »Web 2. $0 \ll^{3}$ - bietet jedermann unzählige Möglichkeiten, "seine Sicht der Dinge« einem weltweiten Publikum unmittelbar zu offenbaren, etwa in Kommentaren zu einem im Internet veröffentlichten Zeitungsartikel, zu einem Blogbeitrag, in einer Mail an eine Mailingliste oder in einer Bewertung auf einem Bewertungsportal (im Folgenden: Internetforen). Dabei ist es nicht unüblich, dass Beiträge unter einem Pseudonym veröffentlicht werden und sich daher die Identität des Autors nicht ohne weiteres bestim-

3 S. hierzu Hilgendorf ZIS 2010, 208, 208 f. 
men lässt. Diese modernen Chancen zur Teilhabe am öffentlichen Diskurs werden indes nicht selten zu sachwidrigen und auch $\mathrm{zu}$ ehrverletzenden Äußerungen missbraucht. Bei internetbasierten Kommunikationsformen liegt zudem eine erhöhte Gefährdungslage vor, die sich psychologisch mit einer wahrgenommenen Anonymität und einer wahrgenommenen Entpersonalisierung erklären lässt. ${ }^{4}$

Wie reagiert die Rechtsordnung hierauf? Materiellrechtlich ist festzuhalten, dass anonymen Äußerungen im Internet im Hinblick auf die Meinungsäußerungsfreiheit derselbe Schutz zuteil wird wie sonstiger Kommunikation (I). Die prozessuale Durchsetzung zivilrechtlicher Ansprüche sowie die strafrechtliche Ahndung von Verstößen wird indes dadurch erschwert, dass zur Aufdeckung der Identität des Autors Internetdienstleister auf dem Wege des Strafprozessrechts in die Pflicht genommen werden müssen (II). Hierbei ist insbesondere bei pressenahen Angeboten im Internet - etwa bei Online-Auftritten von Zeitungen oder bei Blogs - besonderes Augenmerk auf die Verhältnismäßigkeit des Eingriffs zu richten; ein Beschlagnahmeverbot besteht hingegen nicht (III). Abseits der Unterlassungsansprüche (»notice and take down«) ist umstritten, inwieweit auch der Betreiber des Internetdienstes zivilund strafrechtlich zur Verantwortung gezogen werden kann. Die bislang vorherrschende Auffassung, dass eine Vorabkontrolle (»Moderation«) von Beiträgen eines Internetforums dazu führt, dass das Haftungsprivileg der $\S \S 7$ Abs. 2 S. 1, 10 S. 1 TMG entfällt, führt zu regulatorischen Fehlanreizen (IV). Abschließend sei über die Frage des Einsatzes des Strafrechts zu strafrechtsfremden Zwecken nachgedacht (V).

\section{Anonyme Äußerungen im Internet im Lichte der Meinungsfreiheit}

1. a) Das materielle Strafrecht differenziert im Ausgangspunkt zwischen Meinungs- und Tatsachenäußerungen: Meinungsäußerungen, die eine Kundgabe eigener Nichtoder Missachtung darstellen, ${ }^{5}$ sind nach $\S 185$ StGB strafbar, Tatsachenäußerungen, die einen anderen herabwürdigen oder verächtlich machen können und nicht erweislich wahr sind, im Grundsatz ${ }^{6}$ nach $\S 186$ StGB. Zur

4 Vgl. Hilgendorf ZIS 2010, 208, 209ff.; s. auch Heckmann NJW 2012, 2631, 2631; Spindler Gutachten F zum 69. DJT, 2012, F 24.

5 RGSt 40, 416, 416; BGHSt 1, 288, 289; BGHSt 11, 67; st. Rspr.

6 Anders die Kundgabe einer nicht erweislichen Tatsache gegenüber dem Betroffenen, die eine Beleidigung (§ 185 StGB) darstellen kann.
Abgrenzung ist der gesamte Äußerungskontext heranzuziehen; ${ }^{7}$ entscheidend ist, ob die Mitteilung eines Werturteils oder einer Tatsache im Vordergrund steht. ${ }^{8}$ Wenn diese Abgrenzung auch hinsichtlich der Beweislastverteilung und hinsichtlich der in $\S \S 186,187$ und 188 StGB enthaltenen, bei Internetsachverhalten relevanten ${ }^{9}$ Qualifikatione ${ }^{10}$ bedeutsam ist, so wird gerne übersehen, ${ }^{11}$ dass aus verfassungsrechtlicher Sicht auch Tatsachenbehauptungen dem Schutzbereich der Meinungsäußerungsfreiheit (Art. 5 Abs. 1, Abs. 2 GG) unterfallen, »jedenfalls wenn sie Voraussetzung für die Bildung von Meinungen sind «. ${ }^{12}$ Daher muss auch bei $\S 186$ StGB dieser Verfassungsgarantie - etwa im Zusammenspiel mit § 193 StGB - im Sinne der Wechselwirkungslehre zum Durchbruch verholfen werden, insbesondere dann, wenn der Wahrheitsgehalt der Tatsache zum Zeitpunkt ihrer Äußerung ungewiss war: Wenn und soweit eine unbewusst unwahre, nicht beweisbare oder nicht erweislich wahre Tatsache zur Meinungsbildung oder sonst zur Wahrnehmung berechtigter Interessen ${ }^{13}$ beitragen kann, unterliegt auch diese einem verfassungsrechtlichen Schutz. ${ }^{14}$ Jenseits von Schmähkritik und bewusst unwahren Tatsachenbehauptungen ist somit zum Schutze und zur Verwirklichung der Meinungsäußerungsfreiheit des Art. 5 Abs. 1,

7 S. nur BVerfG NJW 2009, 3016 (3018).

8 S. nur BGHSt 6, 159, 162; BGH NJW 1955, 311; Regge/Pegel in: Münchener Kommentar, StGB, 2. Aufl. 2012, § 186 Rdn. 7 ff.

9 Beiträge in öffentlichen Internetforen sind »öffentlich« i.S.d. § 186 StGB, vgl. nur Hilgendorf/Valerius Computer- und Internetstrafrecht, 2. Aufl. 2012, Rdn. 360; MüKo-StGB/Regge/Pegel (Fn. 8) § 186 Rdn. 35. Auf die verbleibenden Konstellationen lässt sich der im Pornografiestrafrecht angewendete, weite Verbreitungsbegriffs - begründet durch BGHSt 47, 55 (58ff.) - auch auf $\S \S 186 \mathrm{ff}$. StGB übertragen, so dass jeweils der höhere Strafrahmen eröffnet ist; a. A. etwa Hilgendorf in: Leipziger Kommentar, 12. Aufl. 2010, § 186 Rdn. 14 a.E.

10 Allein hinsichtlich der Kreditgefährdung stellt § 187 StGB einen eigenständigen Tatbestand dar.

$11 \mathrm{Zu}$ weitgehend daher etwa die Kritik von Lencker/Eisele in: Schönke/Schröder, 28. Aufl. 2010, § 193 Rdn. 15 unter Verweis auf BVerfGE 85, 19 (»Verschiebung der Grenzen von Tatsachenbehauptungen hin zu bloßen Werturteilen«); s. ferner LK/Hilgendorf (Fn. 9) § 185 Rdn. 2. 12 Vgl. nur BVerfGE 85, 23, 31; BVerfGE 94, 1, 7.

13 Etwa im Rahmen einer Strafanzeige: Es ist für eine funktionsfähige Strafrechtspflege unabdingbar, dass sich potentielle Zeugen oder Opfer von Straftaten auch dann an die Strafverfolgungsbehörden wenden, wenn sie die von ihnen behauptete oder für möglich erachtete Straftat nicht beweisen können. S. hierzu BVerfGE 74, 257; Koch NJW 2005, 943.

14 S. nur BVerfGE 61, 1, 8; BVerfGE 85, 1, 17; BVerfGE 90, 241, 247; BVerfGE 94, 1, 7; BVerfGE 114, 339, 352. Allerdings sind hier die von der zivilrechtlichen Rechtsprechung - BGHZ 132, 13; BGH NJW 1987, 2225, 2226 - angenommenen Prüfungspflichten verfassungsrechtlich nicht zu beanstanden, BVerfGE 114, 339, 352. 
Abs. 2 GG eine Gesamtabwägung zwischen den widerstreitenden Positionen der Meinungsfreiheit einerseits und des Persönlichkeitsschutzes andererseits vorzunehmen. ${ }^{15}$

b) Im Rahmen dieser Abwägung sind zunächst sämtliche in Betracht kommenden Interpretationsmöglichkeiten einer Äußerung zu berücksichtigen. ${ }^{16}$ Daher ist auch nicht am Wortsinn oder gar an einer juristischen Fachterminolo-

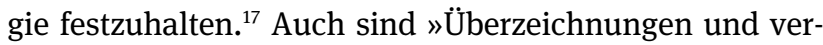
einfachende Verkürzungen ${ }^{18}$ nicht nur im politischen Kontext zulässig. Dem BVerfG zufolge schlage das Pendel dabei umso eher in Richtung Meinungsfreiheit und somit Straflosigkeit aus, »wenn es um Beiträge zum geistigen Meinungskampf in einer die Öffentlichkeit wesentlich berührenden Frage geht«, denn dann »spricht eine Vermutung für die freie Rede «. ${ }^{19}$ Dem ist - entgegen vielstimmiger Kritik in der Literatur ${ }^{20}$ - angesichts der konstitutiven Bedeutung der freien Meinungsäußerung für eine freiheitlich-demokratische Gesellschaft zuzustimmen. (Auch) in diesem Kontext ist nämlich jeglichen $\mathrm{Ge}$ fährdungen entgegenzutreten, die sonst zu übermäßig vorsichtigem, konformem, angepasstem Verhalten führen und eine Mehrzahl von Personen davon abschrecken könnten, von einer Teilhabe am politischen Diskurs aus Furcht vor einer straf- oder zivilrechtlichen Verantwortlichkeit Abstand zu nehmen. ${ }^{21}$ Praktische Konkordanz lässt sich nämlich auch dann erreichen, wenn die beiden verfassungsrechtlichen Güter gewichtet werden und diese Abwägung regelmäßig zu einem Überwiegen einer der beiden Verfassungsgarantien führt. ${ }^{22}$

c) Nach einer Auffassung soll eine anonyme oder pseudonyme Äußerung nur einen geringeren Schutz genießen, da die Meinungsfreiheit eine der Kommunikation und damit dem wechselseitigen Meinungsaustausch - dienende Funktion innehabe, diese aber dann beeinträchtigt

15 Statt vieler BVerfGE 93, 266, 292.

16 BVerfGE 85, 1, 18; BVerfGE 86, 1, 11f.; BVerfGE 93, 266, $295 \mathrm{f}$.

17 S. nur BayObLG NStZ-RR 2002, 40; OLG Frankfurt a. M. NJW 2003, 77 jeweils zum Vorwurf der »Rechtsbeugung «.

18 BGH NJW 1984, 1102, 1103.

19 BVerfG NJW 2009, 3016, 3017 m.w.N.; s. zuvor etwa BVerfGE 93, 266, 303f.; ähnlich auch - für den oben bei und mit Fn. 1 geschilderten Fall - LG Augsburg, Beschl. v. 19. 3. 2013 - 1 Qs 151/13, in diesem Heft, S. 536.

20 Exemplarisch Schönke/Schröder/Lencker/Eisele (Fn. 11) §193 Rdn. 15; LK/Hilgendorf (Fn. 9) § 193 Rdn. 7; jew. m.w.N.

21 Vgl. BVerfGE 65, 1, 43 zum insoweit vergleichbaren Gefühl des Überwacht-Seins.

22 Dies zeigt sich etwa auch im Bereich der Beweisverwertungsverbote, die nur im Ausnahmefall trotz rechtswidrig erhobener oder erlangter Informationen anerkannt werden; s. hierzu BVerfGE 130, 1, $27 \mathrm{ff}$. Zur praktischen Konkordanz zwischen Religionsfreiheit, Ehrenschutz und Meinungsfreiheit s. nur Rox JZ 2013, 30. werde, wenn sich jemand nur unter dem Deckmantel der Anonymität äußere. ${ }^{23}$ Dem kann nicht zugestimmt werden, da es an hinreichenden Gründen fehlt, die eine solche Ungleichbehandlung rechtfertigen könnten: Denn Namen sind ohnehin nur Schall und Rauch. Nicht selten sind bei internetbasierter Kommunikation Fälle anzutreffen, in denen Personen eher über ihr stetig verwendetes Pseudonym identifiziert und adressiert werden denn über deren Klarnamen. Innerhalb eines Internetforums lässt sich auf die Kritik eines unbekannten Autors reagieren und diese - etwa durch Anrede des Kommunikationspartners mit seinem Pseudonym - in Bezug nehmen. Ferner kann die Anonymität die offene, ehrliche Kommunikation jenseits von übermäßiger Konformität oder Angepasstheit fördern. Schließlich ist es in Zeiten, in denen Arbeitgeber regelmäßig im Internet nach Spuren von Bewerbern nachforschen, ${ }^{24}$ gerade für die internetaffinen jungen Generationen von erheblicher Bedeutung, im Internet mit Pseudonymen zu operieren. Daher wäre es auch nicht ausreichend, nur bei ausgeprägten Machtgefällen einen Schutz anonymer Meinungsäußerung anzuerkennen. ${ }^{25}$ Aus diesen Gründen ist daher die Anonymität kein Abwägungskriterium hinsichtlich der Frage, ob eine Äußerung noch von der Meinungsäußerungsfreiheit gedeckt ist. $^{26}$

2. Das Zivilrecht zeichnet - abseits vertraglicher Sonderpflichten ${ }^{27}$ - im Ausgangspunkt die strafrechtlichen und somit inzident auch die verfassungsrechtlichen Wertungen nach, indem es Verletzungen des aus Art. 1, Art. 2 GG folgenden allgemeinen Persönlichkeitsrechts am Maßstab der $\S \S 185,186$ StGB bewertet, die es als Schutzgesetze i.S.d. § 823 Abs. 2 StGB ansieht. ${ }^{28}$ Daneben treten die auf eine Analogie zu § 1004 BGB gestützten Unterlassungsansprüche, die an die Verletzung des allgemeinen Persönlichkeitsrechts anknüpfen und somit ebenfalls auf $\S \S 185$, 186 StGB rekurrieren. ${ }^{29}$ Zwingend ist dies allerdings nicht:

23 Bernreuther, AfP 2011, 218; in diese Richtung auch Beck, MMR 2009, 736, 739, die für anonyme Ehrverletzungen im Internet einen Qualifikationstatbestand fordert.

24 Vgl. hierzu § 32b i. V.m. § 32 Abs. 6 BSDG-E i.d.F. BT-Drs. 17/4230. 25 So aber Bernreuther AfP 2011, 218.

26 In diese Richtung ebenfalls BGH NJW 2009, 2888, 2892; BGH NJW 2007, 2558; OLG Köln NJW-RR 2008, 203, 205; HansOLG Hamburg MMR 2012, 605, 606; Spindler (Fn. 4) F 24.

27 Diese sind etwa bei den Bewertungen im Verkaufsportal eBay einschlägig; s. hierzu LG Köln MMR 2010, 244; LG Düsseldorf MMR 2004, 496; AG Bremen NJW-RR 2010, 1426; AG Koblenz MMR 2004, 638 m. Anm. Ernst; AG Erlangen MMR 2004, 635 sowie Dörre/Kochmann ZUM 2007, 30.

28 S. nur BGHZ 139, 95, 99; BGHZ 99, 133, 136.

29 S. erneut BGHZ 139, 95, 99; BGHZ 99, 133, 136. 
Soweit man das allgemeine Persönlichkeitsrecht als ein sonstiges Rechtsgut i.S.d. § 823 Abs. 1 StGB auffasst, ${ }^{30}$ bestünde auch die Möglichkeit, zivilrechtlich einen weitergehenden Schutz gegen ehrverletzende Äußerungen anzuerkennen als strafrechtlich. Da jedoch die Strafvorschriften der $\S \S 185 \mathrm{ff}$. StGB bereits ausgesprochen weit reichen und das Maß des verfassungsrechtlich Zulässigen vollständig ausschöpfen dürften, ist de lege lata hierfür zwar kein Raum. De lege ferenda spräche viel dafür, die $\S \S 185 \mathrm{ff}$. StGB enger als bisher zu fassen und im Übrigen auf eine zivilrechtliche Rechtsdurchsetzung ${ }^{31}$ zu verweisen.

\section{Zivil- und strafrechtliche Reaktionen gegen den Autor einer anonymen Äußerung - Möglichkeiten und Pflichten zur De-anonymisierung}

1. In beiden Rechtsgebieten besteht die Schwierigkeit, den Urheber der anonymen oder pseudonymen Äußerung zu identifizieren und daher ihn als Klagegegner (im Zivilrecht) bzw. als Beschuldigten (im Strafrecht) zu benennen. Denn die treffsichere und zuverlässige Zuordnung von Anmeldedaten im Internet $\mathrm{zu}$ einzelnen Individuen ist eine seltene Ausnahme. ${ }^{32}$ In aller Regel werden von Benutzern eingegebenen Adress- und Namensdaten allenfalls einer Plausibilitätsprüfung unterzogen; eine verlässliche Identitätsüberprüfung erfolgt nur im Bereich der Finanzdienstleistungen (§4 GwG). Daher ist zur Aufdeckung der Identität einer sich anonym äußernden Person die Rückverfolgung eines konkreten Kommunikationsvorgangs von entscheidender Bedeutung. Als technischer Anhaltspunkt fungiert hierbei die IP-Adresse, die mitsamt einem möglichst exakten Zeitstempel vom Forenbetreiber auf strafprozessualem (2) oder zivilrechtlichem Wege (3) erhoben werden muss. Diese ermöglicht zwar unter Zuhilfenahme des Internetzugangsdienstleisters (4) bestenfalls die Zuordnung zu einem Anschlussinhaber, lässt aber dennoch den Kreis der potentiellen Urheber in aller Regel enger ziehen. Größere Schwierigkeiten bereitet indes die Tatsache, dass IP-Adressen im Endkundenbereich zumeist

30 So die höchstrichterliche Rechtsprechung, vgl. nur BGHZ 161, 266, 268.

31 Ohnehin obliegt die prozessuale Rechtsdurchsetzung bereits jetzt vollständig dem Geschädigten, wenn das Delikt auf den Privatklageweg verwiesen wird; s. hierzu unten II. 5.

32 Ähnlich Weber/Meckbach, NStZ 2006, 492, $492 \mathrm{f}$. dynamisch vergeben werden, aber derzeit deren Zuordnung zu einem Anschlussinhaber nur noch binnen weniger Tage ${ }^{33}$ erfolgen kann.

2. Bei der Erhebung der IP-Adresse nebst Zeitstempel handelt es sich in strafprozessualer Terminologie um die Herausgabe eines Gegenstandes - hier in Form von Daten $^{34}$-, der »für die Untersuchung von Bedeutung sein « kann (§§ 94 Abs. 1, Abs. 2, 95 Abs. 1 StPO). Da in aller Regel der Diensteanbieter die IP-Adresse nicht selbst wahrgenommen hat, sondern diese erst aus seinen Computersystemen auslesen müsste, kommt mangels Vorbereitungs- und Untersuchungspflichten eines Zeugens eine Zeugenaussage nicht in Betracht. Da und soweit die Herausgabe der Daten von einem unverdächtigen Dritten verlangt wird und regelmäßig mit dessen Mitwirkung gerechnet werden kann, ist hierzu somit ein (richterlich anzuordnendes) Auskunftsersuchen bzw. -verlangen zu wählen ( $\S 95$ Abs. 1, 98 Abs. 1 StPO). ${ }^{35}$

Diese Ermittlungsmaßnahme kommt allerdings nur in Betracht, wenn ein (Anfangs-)Verdacht einer strafbaren Handlung vorliegt. Herkömmlich wird ein Anfangsverdacht dann angenommen, wenn es aufgrund kriminalistischer Erfahrungen möglich erscheint, dass eine verfolgbare Straftat begangen wurde. ${ }^{36}$ Auf eine Verurteilungswahrscheinlichkeit komme es - anders als bei dringendem oder hinreichendem Tatverdacht - nicht an. Allerdings ist zu differenzieren: Soweit sich die Ermittlungsbehörden einer unsicheren Tatsachengrundlage ausgesetzt sehen, ist in der Tat zunächst der noch unklare Sachverhalt $\mathrm{zu}$ erforschen. Hinsichtlich der rechtlichen Bewertung hingegen besteht - ähnlich wie im verwaltungsgerichtlichen Eilverfahren ${ }^{37}$ - keine Notwendigkeit, auf eine unsichere prognostische Basis auszuweichen: Bei internetöffentlichen Äußerungen lässt sich Wortlaut und Kontext entweder bereits durch die Anzeigeerstattung oder aber durch einfachste Recherchen ermitteln und auf dieser Grundlage eine Bewertung vornehmen, ob die Äußerung auch unter Berücksichtigung des § 193 StGB und des Art. 5 GG strafrechtlich relevant ist. Nur wenn die Staatsanwaltschaft und der Ermittlungsrichter die Rechtsauffassung vertreten, dass die konkrete Äußerung in ihrem

33 Die IP-Adresse wird oftmals nicht nur bei einem Beitrag eines Benutzers gespeichert, sondern auch bei folgenden Besuchen eines registrierten und angemeldeten Benutzers in einem Internetforum. Daher liefern - jedenfalls bei regelmäßigen Gästen eines Internetforums - auch spätere Besuche eine Spur hin zum Urheber.

34 BVerfGE 113, 29, $50 \mathrm{ff}$.

35 S. zu alledem Brodowski ZIS 2012, 474, 475ff. m.w.N.

36 Statt vieler Meyer-Goßner StPO, 55. Aufl. 2012, § 152 Rdn. 4 m.w.N. 37 Umfassend hierzu Schoch in: Schoch/Schneider/Bier, VwGO, 24. EL 2012, § 80 Rdn. $400 \mathrm{ff}$. 
Kontext strafrechtlich relevant ist, darf daher ein Auskunftsverlangen erlassen werden. Neben dieser Verdachtsschwelle sind bei dem Erlass eines Auskunftsverlangens Beschlagnahmeverbote ( $§ 97$ Abs. 5 StPO $^{38}$ ) im Speziellen und der Grundsatz der Verhältnismäßigkeit im Allgemeinen zu berücksichtigen. Die hierbei vor allem relevanten Aspekte des Presseschutzes bedürfen einer vertieften Betrachtung (unten III).

3. Es ist umstritten, ob auch ein zivilrechtlicher Auskunftsanspruch gegenüber dem Forumsbetreiber besteht. Einer Auffassung nach sei ein solcher nach zivilrechtlichen Grundsätzen gegeben. ${ }^{39}$ Anderer und vorzugswürdiger Auffassung nach entfalten die bußgeldbewehrten und abschließenden datenschutzrechtlichen Beschränkungen (§ 14 Abs. 2 TMG, auch i.V.m. § 15 Abs. 5 S. 3 TMG) eine Sperrwirkung. ${ }^{40}$ Da diese zwar für manche zivilrechtlichen Ansprüche - namentlich »zur Durchsetzung der Rechte am geistigen Eigentum « - eine Ausnahme vorhalten und sich der Gesetzgeber somit der zivilrechtlichen Implikationen dieser Vorschriften bewusst war, kommt eine analoge Anwendung auf die hier maßgeblichen persönlichkeitsrechtlichen Ansprüche nicht in Betracht. Überdies entfiele (jedenfalls ab dem 30. Juni 2013) jede Möglichkeit zur weiteren zivilrechtlichen Zurückverfolgung einer IP-Adresse, für die eine normenklare und normenbestimmte Datenerhebungsbefugnis kraft Verfassungsrecht gefordert ist ${ }^{41}$ und für die eine $\S 101 \mathrm{Abs.} 9$ UrhG vergleichbare Vorschrift fehlt. Daher könnte ein Auskunftsanspruch schon keinen legitimen Zweck verfolgen. Im Übrigen wären auch bei einem zivilrechtlichen Auskunftsanspruch die Verhältnismäßigkeit sowie Auskunftsverweigerungsrechte ${ }^{42} \mathrm{zu}$ beachten.

4. Zur strafprozessualen Zuordnung einer dynamisch vergebenen IP-Adresse ist infolge BVerfGE 130, $151 \mathrm{ab}$ dem 1. Juli 2013 eine gesonderte fachspezifische Eingriffs-

38 Die in einigen Landespressegesetzen noch anzutreffenden Beschlagnahmeverbote bleiben insoweit außer Betracht, da die Regelung der strafprozessualen Zeugnisverweigerungsrechte und Beschlagnahmeverbote die Gesetzgebungskompetenz kompetenzrechtlich dem gerichtlichen Verfahren zuzuordnen sei, von der der Bund insoweit abschließend Gebrauch gemacht habe, BVerfG NJW 1974, 356.

39 OLG Dresden ZD 2012, 388; de lege ferenda Spindler (Fn. 4) F 111f. 40 KG Berlin MMR 2007, 116; OLG Hamm CR 2012, 128; AG München MMR 2011, 417 m. Anm. Müller-Piepenkötter ITRB 2011, 162.

41 BVerfGE 130, 151.

42 Die einfachgesetzliche, presseschützende Vorschrift $\S 383$ Nr. 5 ZPO hinkt der strafprozessualen Rechtsentwicklung jedenfalls seit dem Gesetz zur Änderung der Strafprozessordnung vom 15. Februar 2002 (BGBl 2002 II 682) hinterher und bedarf insoweit einer verfassungsrechtlichen Korrektur, die auch internetbasierten Journalismus erfasst. grundlage erforderlich; der entsprechende Gesetzentwurf zur Einführung eines $\S 100 \mathrm{j}$ StPO befindet sich derzeit noch im Gesetzgebungsverfahren. Die vom Bundestag verabschiedete Fassung ${ }^{43}$ sieht indes keine verfahrensrechtlichen oder materiellen Hürden vor, die über die ohnehin zu beachtende Verhältnismäßigkeit hinausgingen. Die Erfolgsaussichten einer auf $\S 100 \mathrm{j}$ StPO-E gestützten Abfrage ist maßgeblich davon abhängig, wie schnell diese erfolgt und ob zu diesem Zeitpunkt der Dienstleister die Datensätze für eine Zuordnung der dynamisch vergebenen IPAdressen noch vorhält. ${ }^{44}$

5. Ist die Auskunft erteilt worden und konnte der Urheber identifiziert werden, so lohnt sich für einen Geschädigten, der (auch) auf dem Zivilrechtsweg gegen den mutmaßlichen Täter vorgehen möchte, über seinen Rechtsanwalt Akteneinsicht zu beantragen ( $(406$ e Abs. 1 S. 1 StPO). Allerdings ist die Akteneinsicht zu verweigern, wenn überwiegende schutzwürdige Interessen des Beschuldigten oder anderer Personen - wozu auch das Recht auf dessen informationelle Selbstbestimmung zählt - dem entgegensteht. Zieht man hier die Erwägungen aus den sog. Filesharing-Fällen ${ }^{45}$ entsprechend heran, so zeigt sich, dass eine Erheblichkeit der Rechtsverletzung gegeben sein muss, damit Akteneinsicht bewilligt werden kann.

Aus strafprozessualer Sicht ist schließlich daran $\mathrm{zu}$ erinnern, dass es sich bei den Ehrdelikten der $\S \S 185 \mathrm{ff}$. StGB regelmäßig um Privatklagedelikte handelt (§ 374 Nr. 2 StPO), so dass die Staatsanwaltschaft nur bei Vorliegen eines öffentlichen Interesses die Strafverfolgung übernimmt (§ 376 StPO). Ein solches öffentliches Interesse liegt jedoch nicht bereits darin begründet, dass eine Tat internetöffentlich begangen wurde. Hinzutreten muss erstens eine Erheblichkeit der Ehrverletzung ${ }^{46}$ und zweitens eine nicht nur marginale Beeinträchtigung des Rechtsfriedens, der über das Verhältnis zwischen Täter und Opfer hinausreicht. ${ }^{47}$ Das ist jedenfalls dann der Fall, wenn eine unverzügliche Löschung oder Sperrung eines zu Recht beanstandeten Beitrags unterbleibt ${ }^{48}$ oder wenn der beanstandete Beitrag von einer Vielzahl von Personen wahrgenommen wurde. Wurde ein Verfahren auf den Privatklageweg verwiesen, so ist angesichts der mit einer

43 BT-Drs. 17/12879, zuvor BT-Drs. 17/12034.

44 Vgl. hierzu umfassend Sieber, Gutachten C zum 69. DJT, 2012, C $128 \mathrm{ff}$; zur Vorratsdatenspeicherung von Telekommunikations-Verbindungsdaten s. ferner grundlegend BVerfGE 125, 260.

45 Exemplarisch LG Darmstadt K\&R 2009, 211; s. ferner Meyer-Goßner/Schmitt (Fn. 36) § 406e Rdn. 6c m.w.N.

46 Meyer-Goßner (Fn. 36) § 376 Rdn. 1.

47 Valerius, in: Graf (Hrsg.), BeckOK-StPO, 15. Ed. 2012, § 376 Rdn. 2. 48 S. hierzu noch unten IV. 1. 
Privatklage verbundenen Risiken ${ }^{49}$ zur Beschreitung des Zivilrechtsweges $\mathrm{zu}$ raten, der sich - auch finanziell ${ }^{50}-$ lohnen kann.

\section{Aussageverweigerungsrechte von Forenbetreibern und korrespondierende Beschlag- nahmeverbote}

1. a) In der historisch gewachsenen Formulierung der StPO sind gem. $\S 53$ Abs. 1 S. 1 Nr. 5 StPO diejenigen Personen zur Verweigerung des Zeugnisses berechtigt, die in der »Unterrichtung oder Meinungsbildung dienenden Informations- und Kommunikationsdiensten berufsmäßig mitwirken«. Die Sichtweise der StPO ist daher am herkömmlichen Leitbild eines haupt- oder auch nebenberuflich tätigen Journalisten ausgerichtet, wenn auch die Erzielung von Einkünften nicht als Voraussetzung angesehen wird. ${ }^{51}$ Im Internet und hinsichtlich Internetveröffentlichungen sind solche starren beruflichen Grenzen indes verwischt; viele »Blogs« werden als Zusatzangebot zu einer anderen beruflichen (Haupt-)Tätigkeit angeboten oder gänzlich als "Hobby « betrieben. ${ }^{52}$ Doch entscheidend ist betreffend $\S 53$ Abs. 1 S. 1 Nr. 5 StPO ohnehin nicht eine personale, sondern eine funktionale Sicht, ähnlich wie Art. 5 Abs. 1 GG nur am Rande die Presse als Institution, sondern vorrangig die Tätigkeit der Presse schützt. ${ }^{53}$ Daher ist darauf abzustellen, ob sich eine Person durch einen Informationsoder Kommunikationsdienst zumindest mit einem Mindestmaß an Regelmäßigkeit und Beständigkeit an der öf-

49 U.a. Sühneversuch als Prozessvoraussetzung, § 380 Abs. 1 StPO, Einstellungsmöglichkeiten des Gerichts, § 383 Abs. 2 StPO und das Kostenrisiko, §§ 379, 379a StPO.

50 Exemplarisch LG Berlin ZUM 2012, 997: 8. 000 EUR für wiederholte Beleidigungen in sozialen Netzwerken. Indes kann eine solche Zahlung auch dem Täter im Hinblick auf eine Einstellung des Strafverfahrens nach § 153a Abs. 1S. 2 Nr. 1 StPO auferlegt oder als Gegenleistung für die Zurücknahme der Privatklage (§ 391 StPO) vereinbart werden.

51 Vgl. BGH NJW 1999, 2051; Kohlhaas NJW 1958, 41, 41.

52 Dass sich Blogger u.ä. teilweise gegen die Anerkennung als Journalist vor dem Hintergrund sträuben, dass damit auch erhöhte Sorgfaltspflichten verbunden sind (BGHZ 132, 13; BGH NJW 1987, 2225, 2226), vermag an dieser Einschätzung nichts zu ändern. Ob jene Regelungen hinsichtlich Internet-Blogs usw. zeitgemäß und angemessen sind, kann hier indes nicht vertieft werden.

53 So die vorherrschende Argumentationslinie, vgl. etwa BVerfGE 20, 162, 174f.; BVerfGE 66, 116, 134, wenn auch gewisse Schutzpflichten für die Presse bestehen mögen, BVerfGE 80, 124. fentlichen Meinungsbildung inhaltlich beteiligt. Nur im Rahmen dieser funktionellen Tätigkeit ist sodann der personale Schutz des $\S 53$ Abs. 1 S. 1 Nr. 5 StPO eröffnet. Daraus folgt, dass Betreiber von Foren- und Mailinglisten, die ausschließlich fremde Kommunikation vermitteln, sich nicht auf $\S 53$ Abs. 1 S. 1 Nr. 5 StPO berufen können; Blogger und Online-Redakteure von Tageszeitungen hingegen schon.

b) Als zweites Kriterium muss zudem die gesuchte Information privilegiert sein. Da Anmeldedaten und auch die IP-Adresse Informationen sind, die der Identifizierung eines bestimmten Verfassers dienen sollen, ist demzufolge entscheidend, ob der redaktionelle Teil betroffen ist ( $§ 53$ Abs. 1 S. 3 StPO). Auch diese Abgrenzung ist - so die zutreffende Auffassung des BVerfG - nach funktionalen Erwägungen vorzunehmen: Entscheidend sei, dass das Vertrauensverhältnis zwischen Informant und Redaktion geschützt werde, so dass Informanten sich trauen, der Presse diejenigen Informationen zuzuleiten, die »diese zur Wahrnehmung ihrer Kontroll- und Meinungsbildungsfunktion bedarf «. ${ }^{54}$ Daher sei »im Zweifel für die Pressefreiheit « und somit für ein Zeugnisverweigerungsrecht zu entscheiden. ${ }^{55}$ Wenn nun ein Beitrag der Verwirklichung dieser Funktionen diene, so ist er - unabhängig davon, ob er sich im »redaktionellen « oder »Anzeigenteil « eines Presseorgans befindet - durch ein Zeugnisverweigerungsrecht und ein damit korrespondierendes Beschlagnahmeverbot geschützt. ${ }^{56}$ Daher überzeugt es auch, dass die in einer Zeitung veröffentlichten - ggf. anonymisierten - Leserbriefe von $\S 53$ Abs. 1 S. 3 StPO erfasst werden. ${ }^{57}$

2. a) Einer verbreiteten Auffassung zufolge seien Beiträge in einem Internetforum Leserbriefen nicht gleichzustellen, weil diese von jedem eingestellt werden könnten und es einer redaktionellen Selektion oder journalistischredaktionellen Aufarbeitung fehle. ${ }^{58}$ Diese eng am Wortlaut orientierte Argumentation ist vordergründig zutreffend; ihren wahren teleologisch-systematischen Hintergrund kann man indes einer Randbemerkung des $L G$ Oldenburg entnehmen: Dieses wies darauf hin, dass hinsichtlich Leserbriefen »das Presseorgan bzw. der jeweilige Redakteur auch strafrechtlich zur Verantwortung gezogen

54 KG NJW 1984, 1133, 1133 unter Verweis auf BVerfGE 36, 193, 204; BVerfGE 20, 162, 176 und 187.

55 BGHSt [ER] 28, 240, $247 \mathrm{f}$.

56 BVerfGE 64, 108.

57 BVerfGE 64, 108, 114; KG NJW 1984, 1133; Meyer-Goßner/Schmitt (Fn. 36) § 53 Rdn. 40; BeckOK-StPO/Huber (Fn. 47) §53 Rdn. 35.

58 LG Oldenburg NStZ 2011, 655f.; LG Mannheim, Beschl. v. 13. 5. 2005 - 5 Qs 23/05; LG Augsburg, Beschl. v. 19. 3. 2013 - 1 Qs 151/13, in diesem Heft, S. 536; Meyer-Goßner (Fn. 36) § 53 Rdn. 40; Weber/Meckbach NStZ 2006, 492, 493f. 
werden kann, wenn die Grenzen der Pressefreiheit überschritten werden und wenn der Inhalt eines Leserbriefes ... diese Grenzen überschreitet «. ${ }^{59}$ Soweit nämlich der Nachweis individuellen Vorsatzes und individueller Schuld geführt werden kann, können die jeweiligen Redakteure wegen Beihilfe ( $\$ 27$ StGB) zu einem Ehrdelikt bestraft werden.

Doch auch für den Fall, dass eine solche Zurechnung nicht gelingen sollte, haben die meisten Landespressegesetze vorgesorgt, denen zufolge der verantwortliche Redakteur oder der Verleger wegen fahrlässiger Veröffentlichung strafrechtswidriger Inhalte in Presseerzeugnissen bestraft werden kann. ${ }^{60}$ Zusätzlich flankiert wird dies durch strafbewehrte Impressumspflichten ${ }^{61}$ sowie durch ebenfalls strafbewehrte Anforderungen an die Person verantwortlicher Redakteure, u.a. deren unbeschränkte und effektive strafrechtliche Verfolgbarkeit. ${ }^{62}$ Bemerkenswert sind ferner Art. 11 Abs. 2 BayPrG, der »(zu) Lasten des verantwortlichen Redakteurs eines periodischen Druckwerks « die Vermutung aufstellt, »dass er den Inhalt eines unter seiner Verantwortung erschienenen Textes gekannt und den Abdruck gebilligt hat«; ähnlich § 12 HPresseG, der sogar die täterschaftliche Begehung vermutet (es »wird vermutet, dass er die Veröffentlichung ... als eigene Äußerung gewollt hat«) - als fragmentarisch kann man das Pressestrafrecht somit wahrlich nicht bezeichnen. Entlarvend schließlich Art. 11 Abs. 3 S. 2 BayPrG: „Die Bestrafung des Vormanns schließt die des Nachmanns aus«: Wenn der verantwortliche Redakteur den Urheber preisgibt und daher auf sein Zeugnisverweigerungsrecht verzichtet, wird er selbst von strafrechtlicher Verantwortlichkeit frei. ${ }^{63}$

59 LG Oldenburg NStZ 2011, 655, 656.

$60 \S 20$ Abs. 2 LPresseG BW, Art. 11 Abs. 3 S. 1 BayPrG, § 19 Abs. 2 Berliner PresseG, § 14 Abs. 2 BbpPG, § 20 PresseG Bremen, § 19 Abs. 2 Hamburg. PresseG, § 19 Abs. 2 LPrG M-V, § 20 Nds PresseG, § 21 Abs. 2 LPresseG NRW; § 63 Abs. 1 Saarl. MedienG, § 12 Abs. 2 SächsPresseG, $\S 12$ LPresseG S-A, § 14 Abs. 2 LPresseG S-H. Nur das Thüringer PresseG sowie das LMG R-P 2005 enthält keine solche Strafvorschrift (zuvor aber § 19 LPresseG R-P).

61 Exemplarisch $\S 22$ Abs. 1 Nr. 1 i. V.m. § 8 LPresseG BW; strafrechtlich relevant wird dies, sobald es sich um ein »Druckwerk strafbaren Inhalts« handelt (§ 21 Nr. 3 LPresseG BW).

62 Exemplarisch $\S 21$ Abs. 1 Nr. 1 i. V.m. § 9 Abs. 1 Nr. 5 LPresseG BW (»unbeschränkt strafgerichtlich verfolg[bar]«); aus dem Inlandswohnsitzerfordernis (§ 21 Abs. 1 Nr. 1 i.V.m. § 9 Abs. 1 Nr. 1 LPresseG BW) resultiert auch eine faktische Zugriffsmöglichkeit der deutschen Strafjustiz.

63 Ähnlich auch §§ 20, 21 Gesetz über die Presse vom 7. Mai 1874, RGBl. S. 65. Für die zivilrechtliche Haftung »neutraler « Internetdienstleister fordert dies Spindler (Fn. 4), F 113 unter Verweis auf die französische Rechtslage.
Hinter diesen Regelungen steckt mehr als ein archaischer Wunsch, wenigstens irgendjemanden ${ }^{64}$ für eine begangene Ehrverletzung strafrechtlich zur Verantwortung ziehen zu können. Sie sind vielmehr beispielhaft für einen Einsatz des Strafrechts zur Regulierung durch Selbstregulierung, als Mittel hin zur Compliance. Die Presse soll nachdem die Vorzensur entfallen war - unter dem Druck strafrechtlicher Verantwortlichkeit selbst präventiv tätig werden und dafür Sorge tragen, dass die Veröffentlichung strafrechtswidriger Inhalte in ihren Erzeugnissen unterbleibt. Hieraus rechtfertigt sich (auch) das weitgehende, abwägungsfeste Beschlagnahmeverbot des $\S 97$ Abs. 5 S. 1 StPO: Da die Presse selbst ein erhebliches Interesse daran hat, dass sie keine strafbewehrten Beiträge veröffentlicht, erlangt dieses Beschlagnahmeverbot nur in ganz seltenen Fällen betreffend eines Ehrdelikts Bedeutung.

Bei Beiträgen in Internetforen scheitert indes eine solche Compliance, namentlich an $\S \S 7$ Abs. 2 S. 1,10 S. 1 TMG und der Haftungsprivilegierung der Betreiber für fremde, ihnen unbekannte Beiträge. Daher kann hier das Strafrecht auch nicht als Druckmittel hin zu einer Selektion und Vorfilterung der in Foren eingestellten Beiträge wirken. Des Weiteren führte hier ein abwägungsfestes Beschlagnahmeverbot zur faktischen Nichtverfolgbarkeit von einer Vielzahl von Ehrverletzungen im Internet, ${ }^{65}$ das mit dem Ausnahmecharakter von Beschlagnahmeverboten und Zeugnisverweigerungsrechten unvereinbar wäre.

3. Allerdings ist - insbesondere bei pressenahen oder presseähnlichen Internetforen - eine strikte Verhältnismäßigkeitsprüfung vorzunehmen, ${ }^{66}$ die sich nicht in einem bloßen Verweis auf die bereits materiell-rechtlich vorgenommene Abwägung ${ }^{67}$ erschöpfen darf. Zwar handelt es sich bei den Anmeldedaten und der IP-Adresse zumeist um den einzig erfolgversprechenden Ermittlungsansatz und daher um ein legitimes, geeignetes und auch erforderliches Mittel. Im Rahmen der Angemessenheit ist zunächst zu berücksichtigen, dass es sich bei den Beleidigungsdelikten um minder schwere Kriminalitätsformen handelt; andererseits ist aus generalpräventiven Gründen der wahrgenommenen Entpersonalisierung und damit einhergehenden Verrohung der Kommunikationsformen im Internet entgegenzuwirken. Jedoch ist auch zu berücksichtigen, dass sich nicht selten die Autoren, von denen der Ursprungsbeitrag stammt, und mit dem Thema ver-

64 Vgl. Tyler/Mentovich, Punishing Corporate Entities, 19 Journal of Law and Policy (2010) 203, $214 \mathrm{f}$.

65 Weber/Meckbach, NStZ 2006, 492, 494. 66 Weber/Meckbach, NStZ 2006, 492, 495. 67 S. hierzu oben I. 1. 
traute Personen - eventuell gar die zu schützenden Quellen - ebenfalls an der in einem solchen Forum folgenden Diskussion beteiligen. Bei eng mit originär journalistisch-redaktionellen Inhalten verzahnten Internetforen ist diese Nähe und die damit verbundene Gefährdungslage $\mathrm{zu}$ berücksichtigen; allgemein ist $\mathrm{zu}$ beachten, dass die Kommunikation über diese Medien eine bedeutende gesellschaftliche Funktion erfüllt, namentlich zur öffentlichen Meinungsbildung und zur Kontrolle staatlichen und privaten Handelns beiträgt. Ferner ist $\mathrm{zu}$ beachten, dass strafprozessuale Maßnahmen auch über den eigentlichen Ermittlungserfolg hinausgehende Wirkungen haben können. So mögen strafprozessuale Ermittlungsmaßnahmen auch dazu führen, dass Betreiber von Internetforen diese Tätigkeit aufgeben, um nicht wiederholt in derartigen Kontakt mit den Ermittlungsbehörden zu geraten und mit gerichtlichen Beschlüssen konfrontiert zu werden. Sie können ferner dazu führen, dass insbesondere Ängstliche von einer Teilhabe im öffentlichen Diskurs aus der - ggf. irrationalen - Furcht zurückschrecken, selbst Adressat eines Ermittlungsverfahrens zu werden. Angesichts der gebotenen dynamischen Weiterentwicklung des Grundrechtsschutzes ist daher nur bei Ehrdelikten, die in deutlichem Maße die Grenze rechtmäßigen Verhaltens überschreiten, die Angemessenheit einer Auskunftsanordnung zu bejahen. ${ }^{68}$

4. Es ist somit festzuhalten, dass Internetforen und Kommentarfunktionen, die - wie etwa im anfangs genannten Beispiel der Augsburger Allgemeinen Zeitung - mit originär redaktionellen Inhalten verzahnt sind, zwar nicht dem Schutz der $\S \S 33$ Abs. 1 S. 1 Nr. 5, S. 2 u. S. 3, 97 Abs. 5 S. 1 StPO unterliegen, soweit auf Anmeldedaten, IP-Adressen oder sonstige personenbezogene Merkmale zugegriffen werden soll, um die Identität des Urhebers eines anonym eingestellten Beitrags aufzudecken. Allerdings ist die Eingriffshürde der Verhältnismäßigkeit zu beachten, die im Bereich nicht schwerer Ehrverletzungen dazu führt, dass eine Auskunftsanordnung und erst recht ein Beschlagnahme- und Durchsuchungsbeschluss nicht erlassen werden darf.

68 Ähnlich Weber/Meckbach NStZ 2006, 492, 495: Beschlagnahme sei bei »erheblichen Beleidigungsdelikten in Internetforen oftmals verhältnismäßig«.

\section{Die Rechtsstellung des Forenbetreibers: Eine drohende strafrechtliche Fehlregulierung}

1. Der Betreiber eines Internetforums, eines Bewertungsportals oder einer Internet-Mailingliste ist grundsätzlich selbst nicht grundrechtsverpflichtet. Daraus folgt auch, dass der Betreiber im Ausgangspunkt ${ }^{69}$ frei entscheiden kann, wem er Schreib- und Leserechte für sein Angebot einräumt und ob er Kommentare, Meldungen oder E-Mails vorab nach Rechtsverletzungen, nach thematischer Einschlägigkeit oder sogar nach willkürlichen Kriterien auswählt oder diese im Nachhinein löscht. Zudem genießt dieser als sogenannter Hosting-Provider eine Privilegierung: Solange er »keine Kenntnis von der rechtswidrigen Handlung oder der Information« hat, ist er gem. § $10 \mathrm{~S} .1$ Nr. 1 TMG $^{70}$ nicht selbst für diese fremden ${ }^{71}$ Inhalte verantwortlich. ${ }^{72}$ Er ist gem. $§ 7$ Abs. 2 S. 1 TMG auch nicht dazu verpflichtet, Überwachungsmaßnahmen oder stichprobenartige Kontrollen vorzunehmen.

Erlangt er hingegen positive, konkrete ${ }^{73}$ Kenntnis von einem rechtswidrigen, auf seinem Internetangebot gespeicherten Inhalt, so muss er »unverzüglich« tätig werden, »um diese Information zu entfernen oder den Zugang zu ihr zu sperren« (§ 10 S. 1 Nr. 2 TMG). Andernfalls kann er unter Berücksichtigung der herkömmlichen Zurechnungskriterien - für die fortdauernd abrufbare Nachricht strafund zivilrechtlich in Anspruch genommen werden. Hinsichtlich des Kriteriums der »Unverzüglichkeit« knüpft die Rechtsprechung daran an, wie offensichtlich sich die Rechtswidrigkeit aus den dem Diensteanbieter bekannten Informationen erschließt. ${ }^{74}$ Damit wird dem freien Meinungsaustausch Vorrang eingeräumt vor einer vorschnel-

69 Veröffentlichungspflichten sind nur in Ausnahmefällen anzunehmen, etwa im Zuge eines Anspruchs auf Gegendarstellung nach oder entsprechend § 56 Staatsvertrag für Rundfunk und Telemedien; s. hierzu etwa Seitz, in: Hoeren/Sieber, Multimedia-Recht, 32. EL 2012, Teil 8 Rdn. 88.

70 Basierend auf der Richtlinie 2000/31/EG des Europäischen Parlaments und des Rates vom 8. Juni 2000 über bestimmte rechtliche Aspekte der Dienste der Informationsgesellschaft, insbesondere des elektronischen Geschäftsverkehrs, im Binnenmarkt (ABl. EG Nr. L 178 S. 1).

71 Differenzierend hinsichtlich Internetforen einerseits und Bewertungsportalen andererseits Spindler (Fn. 4) F 60 ff.; s. ferner Altenhain, in: Münchener Kommentar, StGB, 1. Aufl. 2010, Vor §§ 7 ff. TMG, Rdn. $23 \mathrm{f}$.

72 Hilgendorf/Valerius (Fn. 9) Rdn. 203.

73 Gercke/Brunst, Praxishandbuch Internetstrafrecht, 2009, Rdn. 600.

74 BGH MMR 2012, 124 m. Anm. Hoeren. 
len Sperrung zweifelhafter Informationen auf unsicherer Tatsachengrundlage; der Diensteanbieter ist jedoch gehalten, ein förmliches Verfahren unter Beteiligung der Betroffenen einzuhalten. ${ }^{75}$

2. a) Fraglich ist allerdings, ob und inwieweit sich ein Betreiber oder Moderator eines Internetforums einer strafoder zivilrechtlichen Haftung aussetzt, der freiwillig eine kostenträchtige Vorfilterung oder eine stichprobenartige Kontrolle der im Forum von Dritten eingestellten Inhalte vornimmt. Diese Vorfilterung ist nämlich - wie jedes menschliches Verhalten - fehlerbehaftet, so dass es trotzdem in derart kontrollierten Foren zu ehrverletzenden Veröffentlichungen kommen kann und auch kommt, etwa weil der Moderator eine strafrechtswidrige Äußerung für noch von der Meinungsfreiheit gedeckt ansieht oder er sich irrigerweise darauf verlässt, dass der Autor eine ehrenrührige Aussage auch beweisen könnte. Einer verbreiteten Ansicht nach kann sich der Betreiber oder Moderator aber in solchen Fällen - weil er durch diese Arbeitsschritte Kenntnis von den Inhalten nimmt - nicht länger auf die Privilegierungen des $\S \S 7,10$ TMG berufen, ${ }^{76}$ da insoweit die Kenntnis der Umstände bereits schadet, ohne dass es auf die Kenntnis der Rechtswidrigkeit ankommt. ${ }^{77} \mathrm{Ihm}$ wird hinsichtlich seiner Beihilfeverantwortlichkeit ${ }^{78}$ allenfalls ein Tatumstands ${ }^{-79}$ oder Verbotsirrtum zugestanden.

Diese Auffassung ist jedenfalls unbillig, da sie mit der Bestrafung desjenigen droht, der Persönlichkeitsrechtsverletzungen im Internet durch eine freiwillig vorgenommene Vorabkontrolle von Beiträgen einzugrenzen versucht. Sie ist auch aus regulatorischer Perspektive verfehlt, denn sie steigert die Anreize, ein Internetforum nicht zu kontrollieren. ${ }^{80}$ Denn nur dann ist der Moderator oder Betreiber vor einer Strafverfolgung wegen - möglicherweise - strafbaren Inhalten geschützt. Doch lässt sich eine Gegenauffassung auch juristisch begründen?

75 BGH MMR 2012, 124, 127.

76 Vgl. etwa Lorenz/Huff (Fn. 2).

77 MüKo-StGB ${ }^{1} /$ Altenhain (Fn. 71) § 10 TMG Rdn. 9 ff. m. umfangr. Nachw., auch zur Gegenauffassung.

78 Zwar hat er als Moderator des Internetforums eine erhebliche Tatherrschaft, da er Nachrichten löschen bzw. sperren kann und er im Rahmen einer Vorfilterung - darüber entscheidet, ob eine Nachricht im Internetforum überhaupt veröffentlicht wird. Allerdings fehlt es ihm in aller Regel sowohl an einem Täterwillen, noch hat er solange er nicht die fremde Nachricht modifiziert - auf den ehrverletzenden Inhalt selbst Einfluss genommen, so dass er lediglich als Gehilfe und nicht als Mittäter anzusehen ist.

79 Die Nichterweislichkeit einer Tatsache bei $\S 186$ StGB ist allerdings eine objektive Bedingung der Strafbarkeit, so dass ein diesbezüglicher Irrtum nicht durch § 16 StGB privilegiert wird, vgl. LK/Vogel (Fn. 9) § 15 Rn. 315.

80 Ähnlich MüKo-StGB'1/Altenhain (Fn. 71) § 10 TMG Rdn. 2. b) Einer verbreiteten Literaturauffassung zufolge ist jedenfalls bei den hier einschlägigen Erfolgsdelikten auch im Rahmen der Beihilfestrafbarkeit die Kausalität der (Beihilfe-)Handlung und deren objektive Zurechnung erforderlich. ${ }^{81}$ Letztere sei nur gegeben, wenn sich durch das Verhalten des Täters T das Risiko eines tatbestandlichen Erfolgseintritts erhöht. Hieraus schließt die vorherrschende Risikoverringerungslehre: ${ }^{22}$ Sinkt durch die Einflussnahme des $\mathrm{T}$ auf einen Kausalverlauf ${ }^{83}$ das Risiko für einund denselben Rechtsgutsträger ${ }^{84}$, so sei der Erfolg dem T nicht zuzurechnen. Als Risiko wird dabei eine Situation verstanden, »in welcher der Eintritt eines Erfolgs (zumindest) mit einer gewissen Wahrscheinlichkeit zu erwarten ist.$^{85}$

Im Rahmen der Risikoverringerungslehre werden nun vorrangig solche Fallkonstellationen diskutiert, in denen die Intensität der drohenden Verletzung - etwa Schulterstatt Kopfverletzung - durch ein Einschreiten des Täters reduziert wird, hingegen das $o b$ der Rechtsgutsverletzung als sicher angenommen wird. Diese Fälle lassen sich allerdings, je nach ihrer konkreten Gestalt, auch über eine fehlende Kausalität einerseits ${ }^{86}$ und eine mutmaßliche Einwilligung ${ }^{87}$ oder durch einen rechtfertigenden Notstand $^{88}$ andererseits zu stimmigen Ergebnissen führen.

In der hier gegebenen Fallgestaltung führen die alternativen Erklärungsansätze für sich genommen nicht weiter, sondern allenfalls die objektive Zurechnung in ihrer Reinform. Der tatsächliche Geschehensablauf ist hierbei mit einem hypothetischen Kausalverlau ${ }^{89} \mathrm{zu}$ vergleichen. Dessen Bestimmung bereitet allerdings Schwierigkeiten: Denkt man die konkrete Moderationshandlung - den »Mausklick« des Moderators - hinweg, so würde die Nachricht nicht veröffentlicht und es käme somit nicht zu einer Intensivierung ${ }^{90}$ der Ehrverletzung. Die Konsequenz dieser Sichtweise wäre, sogar eine Risikoerhöhung durch das Verhalten des Moderators anzunehmen. Vorzugswürdig

81 S. nur Roxin AT II, 2003, § 26 Rdn. 184 m.w.N. 82 So etwa Roxin AT I, 4.Aufl. 2006, §11 Rdn. 53 mit umfangr. Nachw.

83 Andernfalls liegt ein Fall der sog. Risikoersetzung vor, Frisch JuS 2011, 116, 117. In diesen Fällen sei allein auf eine mögliche Rechtfertigung zu rekurrieren, vgl. Kühl AT, 7. Aufl. 2012, § 4 Rdn. 55.

84 Vgl. Kindhäuser ZStW 120 (2008) 481, 495.

85 So zusammenfassend Kindhäuser ZStW 120 (2008) 481, 487.

86 Kindhäuser ZStW 120 (2008) 481, 491 sowie 495.

87 Weber in: Baumann/Weber/Mitsch, StGB, 11.Aufl. 2003, § 14 Rdn. 69; Kindhäuser, ZStW 120 (2008) 481, $496 f$.

88 Kindhäuser, ZStW 120 (2008) 481, 496.

89 So ausdrücklich Roxin (Fn. 82) § 11 Rdn. 61.

90 Die Vollendung der Straftat des Haupttäters tritt nämlich bereits mit der Kenntnisnahme durch den Moderator ein. 
erscheint es, den Arbeitsschritt der Vorfilterung als Ganzes hinwegzudenken: Dann zeigt sich, dass durch die Vorfilterung das Risiko von Ehrverletzungen reduziert wird. Demzufolge schließt eine freiwillig vorgenommene Vorfilterung von Beiträgen in einem Internetforum die objektive Zurechnung aus.

c) Die Rechtsprechung erachtet es jedoch für ausreichend, dass die Haupttat durch die Beihilfehandlung irgendwie gefördert oder erleichtert werde; auf eine Ursächlichkeit - und damit auch auf eine objektive $\mathrm{Zu}$ rechnung - komme es nicht an. ${ }^{91}$ Indes: Auch hier erscheint es zur Bestimmung des Erleichterungs- oder Förderungsmoments geradezu notwendig, einen hypothetischen Kausalverlauf ohne die Beihilfehandlung zu betrachten. Denkt man hier erneut die Vorfilterung weg, so zeigt sich auch hier, dass in der Vornahme einer - im Einzelfall fehlerhaften - Vorfilterung gerade keine Erleichterung oder Förderung der Haupttat zu sehen ist; ex ante erweist sie sich sogar einem potentiellen Täter als Hindernis.

\section{Der Einsatz des Strafrechts zur Verfolgung strafrechtsfremder Zwecke am Beispiel des Pressestrafrechts}

1. Materiell-strafrechtlich zeigte sich bereits, dass die Ehrdelikte im Zusammenspiel mit den Normen des Presserechts hin zu einer strafrechtlichen Compliance wirken, der zufolge Journalisten zur Abwendung einer strafrechtlichen Haftung dazu gehalten sind, eine effektive Vorabkontrolle der unter ihrer Mitwirkung oder Kontrolle veröffentlichten Beiträge vorzunehmen. Das Strafrecht dient hier somit nicht nur der Vergeltung und der Spezial- und Generalprävention, sondern auch dazu, ein System zu schaffen, in dem durch proaktives Tätigwerden Dritter die Begehung bzw. die Folgen von Straftaten zu minimieren sind. Bezogen auf Internetforen droht allerdings eine regulatorische Fehlsteuerung, wenn man nicht eine Vorfilterung von Beiträgen als Risikominimierung und somit als Ausschlussgrund für eine strafrechtliche Verantwortlichkeit ansieht.

2. Doch auch das Strafprozessrecht wird im hier betrachteten Kontext nicht allein zur Durchsetzung des materiellen Strafrechts eingesetzt. In Ermangelung eines zivil-

91 BGH NStZ 2008, 284; vgl. ferner die differenzierten Nachweise bei Fischer StGB, 60. Aufl. 2013, § 27 Rdn. $14 \mathrm{ff.}$ rechtlichen Auskunftsanspruchs ${ }^{92}$ kommt ein Betroffener oftmals nicht umhin, zunächst eine Strafverfolgung $\mathrm{zu}$ initiieren, selbst wenn er nur eine zivilrechtliche Reaktion begehrt oder gar nur an der Aufdeckung der Identität interessiert ist. ${ }^{93}$ Dieser Einsatz des Strafverfahrensrechts zur Verfolgung strafrechtsfremder Zwecke ist indes nicht gänzlich neu; vor Einführung spezifischer urheberrechtlicher Regelungen ( $§ 101$ Abs. 9 UrhG) war dies auch zur Durchsetzung der Rechte am sog. geistigen Eigentum im Internet üblich.

Wie ist diese Entwicklung zu beurteilen? Die Förmlichkeit und Neutralität des strafrechtlichen Verfahrens führen einerseits dazu, dass besonderes Augenmerk auf Verfahrens- und Grundrechte sämtlicher Betroffener gerichtet wird und ausgleichende Elemente des Rechts geradezu zu Wesensmerkmalen des Strafrechts geworden sind - sei es etwa $\S 193$ StGB, sei es das aus der grundgesetzlich geschützten Pressefreiheit resultierende Beschlagnahmeverbot, sei es das Gebot der Verhältnismäßigkeit. Andererseits aber ist die bereits mit einem Ermittlungsverfahren verbundene stigmatisierende Wirkung $\mathrm{zu}$ berücksichtigen; auch werden durch solche Strafverfolgungsmaßnahmen die ohnehin nur begrenzt zur Verfügung stehenden Ressourcen des Kriminaljustizsystems für Ermittlungen gebunden, die sich nur auf Taten mit einem vergleichsweise geringen Unwertgehalt beziehen.

Wenn und soweit sich aber die Grundrechtssensibilität und die Verfahrensrechte aller Betroffenen gleichermaßen auch in anderen Verfahrensordnungen verwirklichen lassen, spricht viel für eine vollständige Verlagerung der Rechtsdurchsetzung ins Zivilrecht durch $\S 101$ Abs. 9 UrhG vergleichbare Vorschriften. Die Rechtsprechung des 1 . Zivilsenats des BGH zu jener Vorschrift lässt jedoch eine ebensolche Sensibilität für grundrechtliche Implikationen und für das Verhältnismäßigkeitsprinzip vermissen, indem sie sich über die klare gesetzgeberische Intention und über die verfassungsrechtlich gebotenen Einschränkungen hinwegsetzt und einen Auskunftsanspruch auch dann annimmt, wenn eine Rechtsverletzungen kein gewerbliches Ausmaß erreicht. ${ }^{94}$

\section{Fazit}

1. Jenseits von Schmähkritik und bewusst unwahren Tatsachenäußerungen ist mit strafrechtlichen wie zivilrecht-

92 S. oben II. 3.

93 So im oben bei und mit Fn. 1 geschilderten Fall.

94 BGH NJW 2012, 2958. 
lichen Mitteln nur vorsichtig tätig zu werden, um den freien Meinungsaustausch - auch auf unsicherer Tatsachengrundlage - weder unmittelbar noch mittelbar zu beeinträchtigen oder auch nur zu gefährden. Daher überzeugt die vom BVerfG aufgestellte »Vermutung für die freie Rede, wenn es um Beiträge zum geistigen Meinungskampf in einer die Öffentlichkeit wesentlich berührenden Frage geht ${ }^{95}$. Der Ehrenschutz tritt - auch unter Berücksichtigung des Gebots praktischer Konkordanz - in diesen Fällen regelmäßig hinter der Meinungsäußerungsfreiheit zurück. Anonyme Äußerungen sind schließlich keine Äußerungen zweiter Klasse, sondern haben in gleichem Maße Anteil am grundgesetzlichen Schutz der Meinungsfreiheit.

2. Da Betreiber von Internetforen, Blogs, Bewertungsportalen usw. nicht selbst grundrechtsverpflichtet sind, lohnt oftmals ein gütlicher Versuch, diese zur Löschung oder Sperrung eines beanstandeten Beitrags zu bewegen, selbst wenn ein Beitrag nicht rechtswidrig sein sollte. Liegt ein rechtswidriger Beitrag vor, so ist der Betreiber nach Kenntnisnahme und pflichtgemäßer Prüfung der Beanstandung verpflichtet, diesen $\mathrm{zu}$ löschen oder zu sperren (»notice and take down«). Eine begrenzte Vorfilterung der Beiträge - etwa durch eine Moderation einer Mailingliste oder eines Forums - führt nicht zu einer Haftung des Betreibers, sondern ist als Risikominimierung aufzufassen, welche die objektive Zurechnung ausschließt bzw. die Haupttat weder fördert noch erleichtert.

3. Zur Durchsetzung der materiellen Rechte lohnt gegenüber dem Urheber einer rechtswidrigen Äußerung zumeist der Zivilrechtsweg. Um einen anonymen Urheber eines Forenbeitrags usw. zu identifizieren, liefert das Zivilrecht allerdings keine hinreichende Handhabe. Jedenfalls für diesen Schritt ist daher ein Ermittlungsverfahren gegen Unbekannt anzustrengen. Eine Auskunftsverpflichtung (§ 95 Abs. 1 StPO), eine Durchsuchung (§ 103 StPO) und eine Beschlagnahme (§ 94 Abs. 2 StPO) gegenüber einem Forenbetreiber unterliegt zwar insoweit nicht dem Beschlagnahmeverbot des $\S 97$ Abs. 5 S. 1 StPO. Allerdings ist eine umfassende rechtliche Prüfung der Strafrechtswidrigkeit der Äußerung und der Verhältnismäßigkeit vorzunehmen. Aus dieser folgt, dass nur bei erheblichen Ehrverletzungen diese prozessualen Maßnahmen ergriffen werden können. 